\title{
Catheter Ablation Versus Medical Therapy for Atrial Fibrillation - Penny wise Pound Foolish !
}

\author{
Gurukripa Kowlgi ${ }^{1}$ and Abhishek Deshmukh ${ }^{1}$ \\ ${ }^{1}$ Mayo Clinic
}

December 4, 2021

\begin{abstract}
Catheter ablation for atrial fibrillation has emerged as a main stay for management of atrial fibrillation. It has been shown to be clinically effective and cost effective in multiple trials .

Catheter Ablation Versus Medical Therapy for Atrial Fibrillation: Penny wise ..Pound Foolish Short Title: Cost-effectiveness of catheter ablation for Atrial Fibrillation

Gurukripa N. Kowlgi, MBBS ${ }^{1}$, Abhishek J. Deshmukh ${ }^{1}$

Department of Cardiovascular Medicine, Mayo Clinic, Rochester, MN, USA
\end{abstract}

Correspondence:

Abhishek Deshmukh, MBBS

Associate Professor of Cardiology

Department of Cardiovascular Medicine

Mayo Clinic, Rochester, MN, USA

E-mail: deshmukh.abhishek@mayo.edu

Phone: 5072551051

Fax: 5072552550

Keywords: Atrial fibrillation, catheter ablation, antiarrhythmic drugs, cost-effectiveness, United Kingdom Funding : None

Disclosures : None

The attributed value of human health is a recurring debate in ethics and medical policy. As such, defining the cost of quality of life (QOL) can be an even harder parameter to quantify as the monetary and QOL perception of pain and suffering can be variable. The task of adding currency values to these intangible elements is a complex yet a fascinating exercise.

Atrial fibrillation (AF) is ubiquitous among patients frequenting utilizing healthcare resources. ${ }^{1} \mathrm{AF}$ is associated with a reduced quality of life (QOL), frequent hospitalizations, increased risk of heart failure (HF), stroke, and mortality. ${ }^{2-4}$ Catheter ablation (CA) has emerged as the mainstay of management with benefits including improvements in QOL, reduced hospitalization, and improved freedom from AF. ${ }^{1,5,6}$ Despite the 
impact of AF on healthcare costs and utilization, there exists a paucity of data in this regard. The present era of healthcare overutilization demands a heightened level of scrutiny towards the cost of care.

In the current issue of the Journal of Cardiovascular Electrophysiology, Leung et al. describe the findings of their elegant study comparing the cost-effectiveness of CA, compared to medical therapy in the United Kingdom. The authors utilize a patient-level Markov health-state transition model for the cost-utility analysis. The utilization data were derived from published data from randomized trials on patients treated for AF with or without concomitant HF. ${ }^{5,7}$ Systematic reviews and meta-analyses were used to gauge the complication rates and recurrences. ${ }^{8,9}$ The publicly available government report on cost in the National Health System was used to formulate costs in the modeled patients. The authors report that CA led to an incremental cost-effectiveness ratio (ICER) of $£ 8,614$ per additional quality-adjusted life-year (QALY) when compared to medical therapy. A similar favorable ICER was seen in simulated cases of AF and concomitant HF. For context, the ICER was compared to a willingness-to-pay (WTP) value of $£ 20,000$ per year of QALY based on the threshold employed by the National Institute of Clinical Excellence (NICE). After a probabilistic sensitivity analysis was applied to 10,000 simulations, CA was found to be cost-effective in $99 \%$ of the cases.

As we veer through the latest and greatest in AF management, it is crucial to take a pause and critically appraise the economic impact of what we have achieved over the last three decades. Leung et al., through their paper, not only summarize the existing data comparing CA and medical therapy for AF but also strive to estimate the costs of each arm in thousands of patient simulations with various permutations. The authors' efforts to shed light on this less-understood topic is praiseworthy indeed. The methodology employed in this article is unambiguous and builds on recent descriptions of CA in AF and HF in the United States and Canada. ${ }^{10,11}$ Our colleagues from across the Atlantic thus add important decibels to the global voice that chants that CA for AF is more cost-effective than medical therapy. Notwithstanding the contrasting medical payment systems and the subjective WTP thresholds in different countries, the conclusion from all the studies are unified and support the cost-effectiveness of CA for AF in patients with or without HF.

The authors exemplify how a cost simulation analysis should be performed with meticulous attention to detail in the model structure. The various possible outcomes of the simulations appear to model a real-world scenario as closely as possible. The model structure includes parallel arms for recurrences and factors in incremental costs of repeat ablations and complications.

The idea of upfront investment to save money down the road is not unique to AF ablation. An apt analogy would be of an owner of an electric car looking to save money on gas in the future. Leung et al. make a strong case that $\mathrm{CA}$ is the "electric car" that a patient suffering from AF deserves!

The authors utilized data from landmark studies in the last 20 years to compute the simulations. As they note, the cost simulation was based on currently used catheters but applied to the clinical findings of studies, including those that used outdated technology. While that likely had an impact, one would anticipate the cost-effectiveness to improve even further if the current clinical outcomes could have been utilized. A similar criticism may be the application of United Kingdom-based cost data to the clinical trials based in the United States. The authors state that the clinical endpoints should not vary dramatically by region. While that point is valid, there may be value in assessing cost-effectiveness across different ethnicities, as racial, gender, comorbidity differences do not solely exist in the clinical presentation, but disparities are fraught even in care delivery. ${ }^{12}$ Understanding that the creation of cost simulation models can be complex, there may have been some degree of oversimplification in the way AF recurrence management was modeled in this study. Leung et al. do well in justifying that limiting cross-over from medical therapy to CA ensures a clear comparison and the lack of modeling more than one CA for recurrence due to lack of published data on multiple ablations. However, despite the superlative efforts in this study, the model simulation does potentially introduce an unavoidable bias that must be appreciated in a critical review.

In summary, Leung et al. have made a valuable contribution to the literature regarding the cost-effectiveness of $\mathrm{CA}$ in $\mathrm{AF}$ as compared to medical therapy. The construct of a willingness-to-pay metric is challenging to grasp with a multitude of variations across countries and continents. Thus, the primary take-home message 
of this paper is the confirmation of findings from similar studies across the world. Despite differences in the money spent on healthcare, and the perception of the value of health, CA appears to be cost-effective compared to medical therapy. Despite the excellent learning points from this paper, when we return to our clinics, we must set our population/financial health suit aside and don the "white coat" for the patient in front of us to ensure the optimum procedure is offered for the appropriate patient.

While we try to be "penny wise" with antiarrhythmic drugs, it is important to not become "pound foolish" with catheter ablation!

\section{REFERENCES:}

1. January CT, Wann LS, Alpert JS, et al. 2014 AHA/ACC/HRS guideline for the management of patients with atrial fibrillation: A report of the American college of Cardiology/American heart association task force on practice guidelines and the heart rhythm society. J Am Coll Cardiol . 2014;64(21):e1-e76. doi:10.1016/j.jacc.2014.03.022

2. Patel NJ, Deshmukh A, Pant S, et al. Contemporary trends of hospitalization for atrial fibrillation in the united states, 2000 through 2010 implications for healthcare planning. Circulation . 2014;129(23):2371-2379. doi:10.1161/CIRCULATIONAHA.114.008201

3. Mehra N, Kowlgi GN, Deshmukh AJ. Predictors of Outcomes in Patients with Atrial Fibrillation: What Can Be Used Now and What Hope Is in the Future. Curr Cardiovasc Risk Rep . 2020;14(9):1-14. doi:10.1007/s12170-020-00645-5

4. Arora S, Jaswaney R, Jani C, et al. Catheter Ablation for Atrial Fibrillation in Patients With Concurrent Heart Failure. Am J Cardiol . 2020;137:45-54. doi:10.1016/J.AMJCARD.2020.09.035

5. Packer DL, Mark DB, Robb RA, et al. Effect of Catheter Ablation vs Antiarrhythmic Drug Therapy on Mortality, Stroke, Bleeding, and Cardiac Arrest among Patients with Atrial Fibrillation: The CABANA Randomized Clinical Trial. In: JAMA - Journal of the American Medical Association. Vol 321. American Medical Association; 2019:1261-1274. doi:10.1001/jama.2019.0693

6. Kowlgi GN, Kapa S. Advances in Atrial Fibrillation Ablation: Energy Sources Here to Stay. Card Electrophysiol Clin . 2020;12(2):167-174. doi:10.1016/j.ccep.2020.02.005

7. Noseworthy PA, Gersh BJ, Kent DM, et al. Atrial fibrillation ablation in practice: assessing CABANA generalizability. Eur Heart J . 2019;40(16):1257-1264. doi:10.1093/EURHEARTJ/EHZ085

8. Noheria A, Kumar A, Wylie J V., Josephson ME. Catheter ablation vs antiarrhythmic drug therapy for atrial fibrillation: a systematic review. Arch Intern Med . 2008;168(6):581-586. doi:10.1001/ARCHINTE.168.6.581

9. Pappone C, Vicedomini G, Augello G, et al. Radiofrequency catheter ablation and antiarrhythmic drug therapy: a prospective, randomized, 4-year follow-up trial: the APAF study. Circ Arrhythm Electrophysiol . 2011;4(6):808-814. doi:10.1161/CIRCEP.111.966408

10. Lau D, Sandhu RK, Andrade JG, Ezekowitz J, So H, Klarenbach S. Cost-utility of catheter ablation for atrial fibrillation in patients with heart failure: An economic evaluation. J Am Heart Assoc . 2021;10(14):19599. doi:10.1161/JAHA.120.019599

11. Chew DS, Loring Z, Anand J, et al. Economic Evaluation of Catheter Ablation of Atrial Fibrillation in Patients with Heart Failure with Reduced Ejection Fraction. Circ Cardiovasc Qual Outcomes . Published online 2020:981-990. doi:10.1161/CIRCOUTCOMES.120.007094

12. Kowlgi GN, Gunda S, Padala SK, Koneru JN, Deshmukh AJ, Ellenbogen KA. Comparison of Frequency of Atrial Fibrillation in Blacks Versus Whites and the Utilization of Race in a Novel Risk Score. Am J Cardiol . 2020;135:68-76. doi:10.1016/j.amjcard.2020.08.029 